\title{
Epilepsias e Gravidez
}

\author{
C.J.R. Campos; ${ }^{*}$ I.C.J. Kairalla**e J.G.C. Lima ${ }^{\star \star \star}$
}

\section{RESUMO}

No presente trabalho săo abordados os diversos fatores discutidos pela literatura mundial no que diz respeito à evolução da gravidez em pacientes epilépticas, tais como hormonais, metabólicos epsicológicos, bem como a influência das complicações gravidicas sobre a freqüência das crises, o controle das epilepsias neste período o os efeitos das drogas anti-epilépticas na mãe e no desenvolvimentofisico e psiquico do feto.

\section{UNITERMOS}

Epilepsias. Gravidez. Homónios. Malformaçōes.

\section{CONSIDERAÇÕES GERAIS}

A história natural das epilepsias pode mostrar flutuações de acordo com a própria síndrome, a faixa etária, a fase de vida da paciente, com incidência variável de diversos fatores desencadeantes e maior ou menor aderência ao tratamento. Essas flutuações podem ocorrer por ocasião de alterações hormonais, mesmo que fisiológicas, como na puberdade, na menstruação, na gravidez e na menopausa.

A freqüência de epilepsia entre mulheres grávidas está por volta de $0,27 \%$, na mesma faixa da população geral e, em aproximadamente $13 \%$ de todos os casos de epilepsia em mulheres grávidas, as crises começaram durante a gravidez (Schmidt, 1982).

Durante a gravidez, pode haver, em cada caso, tanto uma remissão das crises, como uma piora das mesmas, ou mesmo pode o quadro clínico manter-se inalterado. E ainda, como descreve Schmidt (1982), estudos antigos mostravam que o curso das epilepsias era similar numa ampla série de mulheres grávidas tratadas e não tratadas. Rubeska em 1911, apud Remillard e col. (1982), encontrou aumento da frequiência das crises na gravidez em $1 / 3$ dos casos, em 1/3 ela permanecia a mesma e em 1/3 ela diminuia. Já segundo $S c h m i d t$ (1982), em aproximadamente $1 / 4$ dos casos o número de crises aumentava, em 1/4 dos casos diminuía e na metade a frequiência não se alterava.

Atribui-se o aumento das crises durante a gravidez a diversos fatores, tais como: um aumento pré-gestacional na freqüência das crises, a idade materna mais avançada, a maior duração do quadro epiléptico e em multíparas (Remillard e col., 1982).

Há muitos hiatos em nosso conhecimento no que diz respeito à evolução da gravidez numa paciente epiléptica, ao controle da epilepsia nesse período e aos efeitos das drogas anti-epilépticas (DAE) na mãe e no desenvolvimento físico e psíquico do feto. Sabe-se, contudo, que a redução dos níveis séricos das DAE e a não aceitação por parte da gestante da continuidade do tratamento proposto (por receio dos riscos de malformações) parecem ser as principais razões para o aumento na frequêencia das crises, embora os fatores hormonais, metabólicos, respiratórios e psicológicos não possam ser desprezados (Campos, 1986)

Além disso, no que diz respeito ao aspecto medicamentoso, desde que a politerapia tem sido associada a maiores riscos de teratogenicidade, como confirma Lindhout e col. (1984 e 1992a), tem-se apontado para as vantagens da monoterapia, a saber: toxicidade menos freqüente e manuseio mais fácil; eliminação da interação de drogas, a qual parece potencializar os efeitos adversos específicos de cada droga; estimulação da maior aderência da paciente ao tratamento, permitindo ainda, menor custo na aquisição das DAE. Maiores doses e níveis séricos de uma única DAE são em geral de efeito superior no que diz respeito ao sucesso terapêutico, em relação a níveis baixos ou mesmo sub-terapêuticos de múltiplas drogas. 
Os efeitos da gravidez sobre a epilepsia e da epilepsia sobre a gravidez têm sido revisados desde há muitos anos. Apresentaremos, a seguir, cada um dos aspectos citados.

\section{GRAVIDEZ COMO CAUSA DE COMPLICAÇÃO DAS EPILEPSIAS.}

Fatores gestacionais de ordem mais genérica podem interferir na evolução do quadro epiléptico durante a gravidez, quais sejam: complicações da própria gravidez como as náuseas, principalmente matutinas, que podem levar a uma alteração do esquema terapêutico, muitas vezes sem o conhecimento do médico, e ainda a toxemia gravídica, lesões vasculares, embolias, trombose venosa ou raros casos de crescimento de tumor cerebral induzido pela gravidez, como descreve Schmidt (1982).

Para cada caso individual, não é possível se fazer uma previsão da evolução da epilepsia durante a gravidez, devendose buscar apoio para o prognóstico, primeiramente, nos possíveis fatores que modificam a frequêencia das crises na gravidez, segundo Schmidt (1982), como se descreve a seguir:

\section{Fatores metabólicos}

O aumento do volume hídrico durante a gravidez promove uma diminuição da concentração dos íons plasmáticos ( $\mathrm{Na}, \mathrm{K}$, $\mathrm{Mg}, \mathrm{Ca}, \mathrm{Cl}$ ), bem como das DAE, o que pode constituir um dos fatores de aumento das crises neste período. Não se verifica, contudo, uma correlação direta entre a alteração dos níveis séricos das DAE e o aumento ou diminuição do número de crises.

Tem-se encontrado ainda, uma associação de um efeito antidiurético relacionado à dose de carbamazepina (CBZ) . Assim, esta droga pode reduzir os níveis séricos de $\mathrm{Na}, \mathrm{Ca} \mathrm{e} \mathrm{Cl}$, especialmente em pacientes com níveis basais baixos de $\mathrm{Na}$ e altas doses diárias de CBZ (Uhde \& Post, 1983). Contudo, doses baixas de CBZ também podem estar associadas à hiponatremia (Appleby, 1984). O efeito antidiurético da CBZ é acentuado, pelo menos em parte, pela administração concomitante de valproato ou fenobarbital (Sillanpää, 1993). O mecanismo de ação antidiurética é obscuro.

Os registros iniciais de Mygind e col. (1976) e Lander e col. (1977) motivaram inúmeras outras publicaçōes posteriores relatando que a concentração sérica das DAE tende a cair em relação às doses de DAE ingeridas, conforme a gravidez progride (Lander \& Eadie, 1991), com menores níveis séricos no parto e aumentando novamente no período pós-parto, resultando tal declínio, algumas vezes, num aumento da freqüência das crises (Yerby e col.,1992a e 1992b). Contudo, quando as crises estão completamente controladas antes da gravidez, de preferência por um longo tempo, elas quase sempre permanecem completamente controladas durante a gravidez (Lander \& Eadie, 1991).

Segundo Lander e col. (1977), a retenção hídrica e o volume dos tecidos fetais e placentários podem contribuir para a queda da concentração sérica das DAE por aumento dos volumes de distribuição das drogas.

Paralelamente ao declínio na concentração total das DAE durante a gravidez, a fração plasmática livre, não ligada à proteína, parece aumentar progressivamente durante a gravi$\mathrm{dez}$, sendo maior por ocasião do parto. $\mathrm{O}$ aumento da fração livre parece acompanhar a diminuição da concentração de albumina sérica (Yerby e col., 1992a). Estes mesmos autores, já numa publicação anterior (1990), sugerem que a concentração de droga livre seja monitorizada e que os ajustes na dose administrada às pacientes sejam feitos com base na condição clínica das mesmas, na freqüência das crises e na concentração da fração plasmática livre das DAE, sendo que esta última parece permitir um sucesso mais efetivo no controle das crises do que usando valores de concentração total.

Ogawa e col. (1982) não encontraram diferença na duração do trabalho de parto, na perda de sangue durante o parto, no peso da placenta e no peso de nascimento da criança entre as mães epilépticas e as controles normais, enquanto Teramo \& Hiilesmaa (1982) verificaram um discreto aumento no risco de hemorragia durante a gravidez e uma freqüência maior de descolamento prévio de placenta em mulheres epilépticas. Nāo há indícios conclusivos de uma associação possível entre epilepsia e toxemia gravídica, bem como trabalho de parto prematuro não parece ocorrer mais freqüentemente em mulheres epilépticas e também permanecem incertas conclusões a respeito de retardo no crescimento fetal intra-uterino em grávidas epilépticas como se verá mais adiante.

Já para Hill \& Tennyson (1982), as complicações na gravidez de mulheres epilépticas tendem a ocorrer mais freqüentemente que na população em geral, quais sejam: pré-eclâmpsia, hemorragia vaginal no período pré-parto, descolamento de placenta, poli-hidrâmnio, ruptura prematura da bolsa, apresentação anormal do feto e início prematuro do trabalho de parto. Em seus estudos, Hill \& Tennyson (1982) sugeriram que o crescimento intra-uterino pode estar retardado e pode haver maior frequiência de prematuros, o que é confirmado por GöpfertGeyer e col. (1982) que referiram ainda maior frequiência de hemorragia uterina ou convulsão no puerpério.

Ainda segundo Hill (1982), no caso de grávidas epilépticas com níveis séricos baixos de DAE, o médico fica tentado a aumentar a dose das mesmas por medo do aumento das crises durante a gravidez. Contudo, como foi claramente observado no estudo de Ogawa e col. (1982), há passagem livre transplacentária das DAE e, conseqüentemente, o feto ficará exposto a quantidades consideráveis de DAE diretamente relacionadas ao nível sérico materno. Ainda que o fígado do feto e a placenta sejam também capazes de metabolizar as drogas, contribuem, no entanto, num grau menor neste sentido (Teramo \& Hiilesmaa, 1982). Portanto, desde que os níveis tóxicos das DAE para o feto não são conhecidos, sugere-se que devam ser usadas na 
gravidez as menores doses de DAE que conseguirem controlar as crises maternas.

\section{Clearence das DAE na Gravidez}

Os valores máximos de clearence das DAE parecem ocorrer ao redor da ocasião do parto e voltam ao normal poucos dias após o mesmo. Isto fala a favor da necessidade de doses maiores das DAE durante o parto (Teramo \& Hiilesmaa, 1982). Aparentemente há um aumento da função hepática e do metabolismo durante a gravidez o que pode ser uma das explicações para o aumento do clearence. Quanto à função renal, apesar da taxa de filtração glomerular estar aumentada durante a gravidez, a maioria das DAE são eliminadas pela excreção tubular que não está modificada nesta fase (Teramo \& Hiilesmaa, 1982). Mygind e col. (1976) concluiram em seus estudos, que o clearence renal aumentado poderia ser explicado pelas alterações nas ligações proteicas das DAE que ocorrem nesta fase.

\section{Mal-absorção das DAE}

A composição do suco gástrico está modificada na gravidez, resultando num $\mathrm{pH}$ gástrico maior e no aumento da capacidade de tamponamento. A motilidade diminuída e a alteração da fraca ionização ácida ou básica das drogas pođeriam influenciar as taxas de absorção (Teramo \& Hiilesmaa, 1982). Contudo, esta questão ainda não se encontra totalmente esclarecida (Yerby e col., 1992a).

\section{Influências hormonais}

Tem sido demonstrado que os estrógenos parecem ter ação epileptogênica e a progesterona efeito oposto, uma vez que o estrógeno pode diminuir os níveis das DAE por indução da atividade enzimática dos microssomos hepáticos e, deste modo, aumentar a frequiência das crises (Bag e col., 1989). E ainda, segundo Teramo \& Hiilesmaa (1982), as drogas parecem competir com a progesterona e o estrogênio nos sítios de ligação enzimática durante a gravidez, o que torna o quadro mais complexo. Existem, por outro lado, efeitos específicos concomitantes à geração das crises, como mostram os estudos experimentais de Amado e col.(1993).

\section{Fatores Psicológicos}

O psiquismo da mulher na gestação se modifica, com tendência tanto a uma hipersusceptibilidade aos estímulos, como a uma sensação de bem estar subjetivo, talvez relacionada com a elevação do nível de endorfinemia. Essa mudança no psiquismo pode levar a um novo enfoque da epilepsia e suas repercussões sobre a vida da paciente, com resultados imprevisíveis sobre a frequêencia das crises. As tensões psíquicas durante a gravidez podem desencadear insônia que por sua vez pode acentuar essas tensões, as quais, então, poderão desencadear fadiga, hiperventilação, quadros psicossomáticos que poderão, por sua vez, transformar-se em fatores desencadeantes de crises epilépticas. Além disto, o medo do risco de dar a luz a um filho epiléptico ou malformado pode, por si só, constituir-se também num fator desencadeante das crises ou pode levar a gestante à não-aderência ao tratamento medicamentoso $\mathrm{e}$, com isto, contribuir para o decréscimo das concentrações séricas das DAE e subseqüente aumento das crises. Contudo, inversamente, a aderência ao tratamento pode aumentar, caso a mulher fique preocupada com o risco das crises afetarem a saúde do feto.

\section{CRISES EPILEPTICAS COMO CAUSA DE COMPLICAÇÃO DA GRAVIDEZ.}

Crises convulsivas generalizadas têm sido associadas com aumento do risco de aborto. Porém, crises de todos os tipos podem estar relacionadas com maior risco de acidentes ou hipóxia. E ainda, as crises recorrentes sem controle apropriado estão associadas com declínio no desempenho das funções neuropsicológicas dos filhos de mães epilépticas (Yerby e col., 1990).

A complicação mais séria da epilepsia para a mãe e o feto é o status epilepticus. Esta é uma complicação rara, mas quando ocorre, representa risco de vida, tanto para a mãe quanto para o feto ou o recém-nascido. É uma condição que pode ocorrer repentinamente ou ser subseqüente a um aumento gradativo no número e gravidade das crises. O status epilepticus pode ocorrer a qualquer tempo durante a gravidez, o trabalho de parto e o puerpério. A mortalidade para a mãe e a criança é alta. Se a criança nasce viva, ela pode morrer em poucos dias por prematuridade ou depressão respiratória e do Sistema Nervoso Central pelas drogas usadas para tratar a mãe (Hill \& Tennyson, 1982). Há até mesmo quem recomende interromper a gravidez como único meio de controlar o status, embora tenha sido relatado o caso de uma gestante com status epilepticus onde mãe e filho sobreviveram (Goodwin \& Lawson, 1947).

\section{MALFORMAÇÕES CONGENITAS}

Mulheres epilépticas tendem a apresentar um alto risco gestacional e obstétrico. Além disso, outros fatores podem também ter um efeito adverso no recém-nascido, como descreve van der Pol e col. (1991), como por exemplo um status sócio-econômico baixo e uma frequiência maior de doenças familiares e anomalias congênitas. Estas últimas, por sua vez, são descritas em função de diversas variáveis que tornam complexos os estudos sobre as malformações congênitas major e minor em filhos de mães epilépticas, a saber: possíveis efeitos teratogênicos das DAE, o caráter endógeno das epilepsias com relação ao seu potencial teratogênico e a pressuposição de que as crises possam ter efeito deletério sobre o feto (Berzaghi e 
col., 1990; van der Pol e col., 1991), muito embora não esteja estabelecido definitivamente se as crianças de mães que convulsionam durante a gravidez têm maior risco de malformações que aquelas de mães sem crises. Num estudo prospectivo de coorte, nem a presença ou a ausência de crises maternas durante a gravidez e nem o tipo de crise teve qualquer impacto sobre o número de anomalias minor por criança (Yerby e col., 1992b).

Tem sido relatado que filhos de mães epilépticas apresentam malformações congênitas 2,5 vezes mais que a população em geral (Pellock, 1989). Fedrick (1973) fez um levantamento dos arquivos do Oxford Record Linkage Study, encontrando um contingente mais alto de anormalidades congênitas entre crianças nascidas de mães epilépticas $(13,8 \%)$ quando comparadas com grupos controles $(5,6 \%)$. Esse mesmo autor notou que nem a frequiência de crises e nem o período de duração da epilepsia da mãe pareciam ter qualquer relação com a frequiência de defeitos do recém-nascido, com excessão de 2 mães que desenvolveram epilepsia no $1^{\circ}$ trimestre de gravidez. A ocorrência de crises no $1^{\circ}$ trimestre de gravidez tem sido associada a índices maiores de malformaçōes $(12,3 \%$ contra $4 \%)$ sem preferência para um tipo específico de crise (Lindhout e col., 1992a). Nakane e col. (1980), num estudo de colaboração multi-institucional no Japão, encontraram uma frequiência de malformação fetal de $2,3 \%$ num grupo não medicado e $11,5 \%$ no grupo medicado.

A Síndrome fetal que pode ocorrer em consequência do uso das DAE é um cluster de malformações envolvendo anormalidades dos lábios ( 3 a 10 vezes mais freqüentes entre crianças de mães epilépticas), do pálato, face, crânio, do tubo neural, além de anormalidades cardiovascular ( 3 a 8 vezes mais comum em crianças de mães epilépticas em tratamento), do crescimento e do desenvolvimento (Lander \& Eadie, 1991).

Yerby e col. (1992b) chamaram atenção para as chamadas "anomalias estruturais minor" relacionadas às DAE e que não constituem uma ameaça à saúde, ocorrendo em menos de $4 \%$ da população. Segundo Janz (1982), as malformações minor mais frequientes associadas às epilepsias incluem displasia facial e hipoplasia dos dedos das mãos e dos pés, enquanto as major incluem doença cardíaca congênita e malformação pálato-labial.

A ocorrência de microcefalia associada ao uso de DAE, bem como o retardo no crescimento pré-natal e pós-natal e o menor ganho de pêso no $1^{\circ}$ mês pós-natal parecem ser um achado discutível, na medida em que são algumas vezes transitórios e causas genéticas parecem contribuir para tais fatos, como demonstraram Gaily \& Granström (1989) e Gaily e col. (1990). Os efeitos sedativos das drogas provavelmente também explicam, pelo menos parcialmente, o deficit transitório de peso por um aleitamento sub-ótimo. E ainda, com relação à diferença de estatura entre essas crianças e o grupo controle, estes mesmos autores sugeriram que a estatura dos pais pareceu ser o fator preponderante a influenciar o deficit de estatura dessas crianças. Os exames clínicos, portanto, deveriam sempre incluir a mensuração do perímetro cefálico e da estatura parentais para permitir uma estima- tiva dos efeitos isolados da hereditariedade e da exposição às DAE.

Com relação às DAE utilizadas, Fedrick (1973) refere que a fenitoína parece provocar, de longe, maior número de defeitos que o fenobarbital, mas se as 2 drogas forem ingeridas conjuntamente, o efeito é ainda mais pronunciado. Tem-se discutido ainda, a associação entre o uso de valproato e/ou CBZ e a ocorrência de espinha bífida ou malformações do tubo neural (Lindhout e col., 1992a e b). Jones e col. (1989) demonstraram a ocorrência de anomalias minor com o uso de CBZ.

Uma vez que o risco de malformação congênita em filhos de mãe epiléptica é freqüentemente causa de preocupação, Firth \& Lindenbaum (1989) consideraram ser melhor ingerir uma droga como o valproato de sódio e aceitar um risco maior de defeito no tubo neural, que pode ser precisamente diagnosticado em fases precoces da gravidez por meio da dosagem de alfa-fetoproteína no soro materno e registros de ultrassom de alta resolução, e com isto permitir providências adequadas logo após o nascimento no sentido do devido apoio terapêtico à criança e psicológico aos pais, do que ingerir uma outra droga e correr um risco substancial, talvez de $20 \%$, de atraso no desenvolvimento, que nenhum teste pré-natal é capaz de avaliar, o que talvez só fosse acabar sendo detectado em fases - tardias, impedindo condutas adequadas de tratamento preventivo.

\section{CONCLUSÕES}

Como se pôde ver, os dados obtidos em diversos estudos não se mostram conclusivos. Os resultados variam de autor para autor, sendo as diferenças na população de pacientes, no manejo e no desenho dos estudos, possivelmente as responsáveis, pelo menos em parte, por essas variações.

De qualquer modo, para que possamos estabelecer a melhor conduta para as epilepsias na gravidez, é fundamental que se tenha tanto um diagnóstico preciso em relação aos tipos de crises epilépticas, às síndromes epilépticas e suas possíveis etiologias, bem como um conhecimento das propriedades farmacocinéticas e farmacodinâmicas das DAE escolhidas, bem como também se faça o diagnóstico diferencial com crises de outras origens, como a lipotimia e a síncope, bastante comuns na gravidez. As pseudo-crises de natureza histérica podem também se associar às crises verdadeiras, dificultando a interpretação dos resultados do tratamento.

Parece haver uma tendência a um controle melhor das crises durante a gravidez naqueles casos em que já se conseguiu um controle efetivo, de preferência com monoterapia, previamente à ocorrência da mesma. Assim, esses aspectos devem ser ajustados antes da gravidez, a fim de se alcançar o controle ótimo das crises. Porém, não há fatores preditivos a partir dos quais se possa antecipar um curso individual das epilepsias durante a gravidez. 
A primeira crise convulsiva, ou crise isolada, em pacientes com ou sem antecedentes de crises epilépticas, deve ser considerada inicialmente como sintomática, devendo-se investigar, portanto, um possível processo mórbido atual (distúrbios metabólicos, acidente vascular cerebral isquêmico, tromboflebites cerebrais, meningites, tumores e eclâmpsia). Quando for feito o diagnóstico de um processo ativo atual, o tratamento específico deve ser instituído.

Com relação aos estudos sobre a ocorrência de malformações major e minor em filhos de pacientes grávidas epilépticas, devemos, antes de mais nada, obter informações corretas no que diz respeito à etiologia da epilepsia, tipo e ocorrência de crises durante a gravidez e, então, o tipo de DAE usada, dose total e dose máxima diária prescrita no $1^{\circ}$ trimestre da gravidez. Com esses dados, estaremos em melhor posição de determinar os efeitos nocivos e teratogênicos específicos de cada droga, lembrando sempre que parece haver uma superposição dos efeitos das drogas sobre os fatores endógenos hereditários.

O fenobarbital em monoterapia tem sido mais preconizado em nosso meio como tratamento sintomático para as crises epilépticas durante a gravidez (Campos, 1986), embora o potencial teratogênico das DAE seja um problema complexo, onde vários fatores entram em jogo concomitantemente, como a politerapia, o caráter endógeno das epilepsias, as crises em si e as intercorrências obstétricas associadas.

Desde que nenhuma droga específica e nenhuma dose terapêutica provou ser claramente de maior risco para o feto, pode não ser justificada durante a gravidez uma mudança na medicação ou a redução da dose terapêutica em uso (Janz, 1982), embora se possa recomendar sempre as menores doses de DAE que já tenham controlado anteriormente as crises da paciente, uma vez que não são conhecidos com clareza quais os níveis tóxicos para o feto (Hill, 1992).

A razão do atraso no crescimento de crianças nascidas de mães epilépticas, bem como a redução do perímetro cefálico, tem sido discutida, parecendo, no primeiro caso, ser um fenômeno transitório, ou então, em ambos os casos, admite-se mais um caráter prioritariamente genético, se consideradas as medidas das figuras parentais, do que uma consequiência da influência das DAEs utilizadas por suas mães (Gaily \& Granström, 1989; Gaily e col., 1990).

Para terminar, podemos dizer que quando lidamos com uma paciente epiléptica que está grávida ou planejando engravidar, várias são as medidas potencialmente benéficas que podem ser adotadas:

a) considerar a gravidez como de alto risco e manter seguimento neurológico e obstétrico rigoroso;

b) evitar ganhos excessivos de peso e retenção hídrica, que podem levar a um aumento de frequiência de crises;

c) sempre que possível colocar a paciente sob tratamento monoterápico antes da gravidez, associando-se uma revisão cuidadosa dos fatores de risco em cada caso e usando-se a droga supostamente menos teratogênica (costuma-se dar preferência ao fenobarbital) e com a menor dose efetiva.
Nesse caso é valiosa a ajuda da monitorização dos níveis séricos de drogas antiepilépticas.

d) torna-se conveniente um follow-up especializado e cuidadoso, suporte nutricional e suplemento vitamínico apropriado, particularmente o folato, ainda que isso possa ser questionável.

e) prevenir as hemorragias potenciais do $3^{\circ}$ trimestre e do neonato, provocadas pelo uso de fenitoína, fenobarbital ou primidona. Essas drogas podem provocar uma hipoprotrombinemia e uma depressão dos fatores de coagulação dependentes de vitamina $K$, tendo sido sua administração preventiva preconizada durante $o 3^{\circ}$ trimestre da gravidez, no início do trabalho de parto e no nascimento (Pellock, 1989).

f) com relação ao receio de transmissão genética da epilepsia para o filho, devemos orientar a paciente no sentido de que, embora exista fator hereditário, o fator adquirido é, sem dúvida, aquele que tem sido enfatizado como de maior importância na etiologia das epilepsias, mesmo naqueles quadros descritos como tipicamente genéticos (van der Pol, 1991). Na verdade, talvez seja sempre necessário um fator ambiental para que haja manifestações clínicas das epilepsias. Dessa forma, o medo de que o filho a ser gerado possa padecer de problema igual ao seu diminui, deixando a paciente mais tranqüila e menos propensa a crises.

\section{SUMMARY}

Several factors presented in the world literature are discussed in this paper, conceming to the evolution of the pregnancy in epileptic patients, such as hormones, methabolic and psychologic events, as well the influence of the pregnancy complications on the crises frequency, the control of the epilepsy in this period and the effects of the anti-epileptic drugs on the mother and on the physical and psychical fetus development.

\section{KEY WORDS}

Epilepsies. Pregnancy. Hormones. Malformations.

\section{Bibliografia}

1. AMADO, D.; CAVALHEIRO, E.A.; BENTIVOGLIO, M. - Epilepsy and hormonal regulation: the patterns of $\mathrm{GnRH}$ and galanin immunoreactivity in the hypothalamus of epileptic female rats. Epilepsy Research 14: 149-159, 1993.

2. APPLEBY, L. - Rapid development of hyponatremia during low dose carbamazepine therapy. J. Neurol. Neurosurg. Psychlatry 47: 1138,1984

3. BAG, S.; BEHARI, M.; AHUJA, G.K.; KARMARKAR, M.G. Pregnancy and epilepsy. J. Neurol. 236 (5): 311-313, 1989.

4. BERZAGHI, M.P.P.; NAFFAH-MAZZACORATTI, M.G.; AMADO, D.; CAVALHEIRO, E.A. - Effect of amygdalid kindled seizures during pregnancy on neonatal brain biogenic amines. Brazilian J. Med. Biol. Res. 23:827-30, 1990.

5. CAMPOS, C.J.R. - Epilepsias e gravidez. Rev. Bras. Clin. Terap. 15(5): 129-134, 1986.

6. FEDRICK, J. - Epilepsy and Pregnancy: A report from the Oxford Record Linkage Study. Br. Med. J. 2: 442-8, 1973. 
7. FIRTH, H.V. \& LINDENBAUM, R.H. - Epilepsy in women of childbearing age. Br. Med. J. 299 (6705): 976-977, 1989.

8. GAILY,E. \& GRANSTRÖM, M.L. - A transient retardation of early postnatal growth in drug-exposed children of epileptic mothers. Epilepsy Res. 4: 147-155, 1989.

9. GAILY, E.K.; GRANSTRÖM, M.L.; HIILESMAA, V.K.;BARDY, A.H.. Head circumference in children of epileptic mothers: contributions of drug exposure and genetic background. Epilepsy Res. 5: 217-222, 1990.

10. GOODWIN, J.F. \& LAWSON, C.W. - Status epilepticus complicating pregnancy. Br. Med. J. 2: 332-3, 1947.

11. GÖPFERT-GEYER, I.; KOCH, S.; RATING, D.; JÄGER-ROMAN, E.; HARTMANN, A.; JACOB, S.; OFFERMANN, G.; HELGE, H. Delivery, Gestation, Data at Birth, and Neonatal Period in Children of Epileptic Mothers. In: JANZ, D.; DAM, M.; RICHENS, A.; BOSSI, L.; HELGE, H.; SCHMIDT, D. Epilepsy, Pregnancy and the Child. New York, Raven Press, 1982. p. 3-14.

12. HILL, R.M. - The Advisability of Increasing Antiepileptic Drug Dosage During Pregnancy. In: JANZ, D.; DAM, M.; RICHENS, A.; BOSSl, L.; HELGE, H.; SCHMIDT, D. Epilepsy, Pregnancy and the Child. New York, Raven Press, 1982. p. 163.

13. HILL, R.M. \& TENNYSON, L. - Premature Delivery, Gestational Age, Complications of Delivery, Vital Data at Birth on Newborn Infants of Epileptic Mothers: Review of the Literature. In: JANZ, D.; DAM, M.; RICHENS, A.; BOSSI, L.; HELGE, H.; SCHMIDT, D. Epilepsy, Pregnancy and the Child. New York, Raven Press, 1982. p. 167-73.

14. JANZ, D. - On Major Malformations and Minor Anomalies in the Offspring of Parents with Epilepsy: Review of the Literature. In: JANZ, D.; DAM, M.; RICHENS, A.; BOSSI, L.; HELGE, H.; SCHMIDT, D. Epilepsy, Pregnancy and the Child. New York, Raven Press, 1982. p. 211-22.

15. JONES, K.L.; LACRO, R.V.; JOHNSON, K.A.; ADAMS, J. - Pattern of Malformations in the Children of Womem treated with Carbamazepine during pregnancy. The New England Journal of Medicine, 320: 1661-6, 1989.

16. LANDER, C.M.; EDWARDS, V.E.; EADIE, M.J.; TYRER, J.H. Plasma anticonvulsant concentrations during pregnancy. Neurology 27: 128-31, 1977.

17. LANDER, C.M. \& EADIE, M.J. - Plasma Antiepileptic Drug Concentrations During Pregnancy. Epilepsla 32(2): 257-266, 1991.

18. LINDHOUT,D.; HÖPPENER, R.J.E.A.; MEINARDI, H. Teratogenicity of Antiepileptic Drug Combinations with Special Emphasis on Epoxidation (of Carbamazepine). Epilepsia 25(1): 77-83, 1984.

19. LINDHOUT, D.; MEINARDI, H.; MEIJER, J.W.A.; NAU, H. Antiepileptic drugs and teratogenesis in two consecutive cohorts: Changes in prescription policy paralleled by changes in pattern of malformations. Neurology 42 (Suppl.5): $94-110,1992 a$.

20. LINDHOUT,D.; OMTZIGT, J.G.C.; CORNEL, M.C. - Spectrum of neural-tube defects in 34 infants prenatally exposed to antiepileptic drugs. Neurology 42 (Suppl.5): 111-118, 1992b.

21. MYGIND, K.I.; DAM, M.; CHRISTIANSEN, J. - Phenytoin and Phenobarbitone Plasma Clearence during Pregnancy. Acta Neurol. Scandinav. 54: 160-66, 1976.

22. NAKANE, Y. $\theta$ col. - Multi-institutional Study on the Teratogenicity and Fetal Toxicity of Antiepileptic Drugs: A Report of a Collaborative Study Group in Japan. Epllepsia 21: 663-80, 1980.
23. OGAWA, Y.; NOMURA, Y.; KANEKO, S.; SUZUKI, K.; SATO, T. Insidious Effect of Antiepileptic Drugs in the Perinatal Period. In: JANZ,D.; DAM, M.; RICHENS, A.; BOSSI, L.; HELGE, H.; SCHMIDT, D. Epilepsy, Pregnancy and the Chlld. New York, Raven Press, 1982. p. 197-202.

24. PELLOCK, J.M. - Efficacy and Adverse Effects of Antiepileptic Drugs. Pedlatric Clinics of North America 36 (2): 435-448, 1989.

25. REMILLARD, G.; DANSKY, L.; ANDERMANN, E.; ANDERMANN, F. - Seizure Frequency During Pregnancy and the Puerprium. In: JANZ, D.; DAM, M.; RICHENS, A.; BOSSI, L.; HELGE, H.; SCHMIDT, D. Epilepsy, Pregnancy and the Child. New York, Raven Press, 1982. p. 15-26.

26. SCHMIDT, D. - The Effect of Pregnancy on the Natural History of Epilepsy: Review of the Literature. In: JANZ, D.; DAM, M.; RICHENS, A.; BOSSI, L.; HELGE, H.; SCHMIDT, D. Epilepsy, Pregnancy and the Child. New York, Raven Press, 1982. p. 3-14.

27. SILLANPÄÄ, M. - Carbamazepine. In: WYLLIE, E.. The Treatment of Epilepsy - Principles and Practice. Philadelphia, Lea \& Febiger, 1993. p. 867-886.

28. TERAMO, K. \& HIILESMAA, V.K. - Pregnancy and Fetal Complications in Epileptic Pregnancies: Review of the Literature. In: JANZ, D.; DAM, M.; RICHENS, A.; BOSSI, L.; HELGE, H.; SCHMIDT, D. Epilepsy, Pregnancy and the Child. New York Raven Press, 1982. p. 53-59.

29. UHDE, T.W. \& POST, R.M. - Effects of carbamazepine on serum electrolytes: clinical and theoretical implications. J. Clin. Psychopharmacol. 3: 103-106, 1983.

30. VAN DER POL, M.C.; HADDERS-ALGRA, M.; HUISJES, H.J. TOUWEN, B.C.L. - Antiepileptic medication in pregnancy: Late effects on the children's central nervous system development. Am. J. Obstet. Gynecol. 164 (1-part 1): 121-128, 1991.

31. YERBY, M.S.; FRIEL, P.N.; MCCORMICK, K.; KOERNER, M.; VAN ALLEN, M.; LEAVITT, A.M.; SELLS, C.J.; YERBY, J.A.. Pharmacokinetics of anticonvulsivants in pregnancy: alterations in plasma protein binding. Epilepsy Res. 5: 223-228, 1990.

32. YERBY, M.S.; FRIEL, P.N.; MCCORMICK, K. - Antiepileptic drug disposition during pregnancy. Neurology 42(Suppl.5): 12-16, 1992a.

33. YERBY, M.S.; LEAVIT, A.; ERICKSON, D.M.; MCCORMICK, K.B.; LOEWENSON, R.B.; SELLS,C.J.; BENEDETTI, T.J. Antiepileptics and the development of congenital anomalies. Neurology 42 (Suppl.5): 132-140, $1992 \mathrm{~b}$.

\section{Nota dos autores:}

ALERTA - Recentemente foi recomendado pelo FDA e pelo fabricante a interrupção da prescrição do medicamento antiepiléptico FELBATOL (felbamato), apesar de sua efetividade em alguns casos de epilepsia de difícil controle. Isto foi devido a relato de casos fatais de anemia aplásica ligados ao uso dessa droga.

\section{Correspondêncla}

Prof. Dr. C.J.R. Campos

Setor de Investigação e Tratamento das Epilepsias

Disciplina de Neurologia da EPM. - Rua Pedro de Toledo, 655

Săo Paulo, SP., Brasil. CEP 04039-030 